\title{
Correction to: Development of Apremilast Solid Dispersion Using TPGS and PVPVA with Enhanced Solubility and Bioavailability
}

\author{
Liuhong Yang, ${ }^{1}$ Penghui Wu, ${ }^{1}$ Jinchao Xu, ${ }^{1}$ Dihuan Xie, ${ }^{1}$ Zhongqing Wang, ${ }^{1}$ Qian Wang, ${ }^{1}$ Yong Chen, ${ }^{1,2,5}$ \\ Chuan Hua Li, ${ }^{2}$ Jiaxin Zhang, ${ }^{3}$ Hangping Chen, ${ }^{4}$ and Guilan Quan ${ }^{4,5}$
}

published online 14 June 2021

\section{Correction to: AAPS PharmSciTech https://doi.org/10.1208/s12249-021-02005-x}

This correction is to add the additional corresponding email address quanguilan@jnu.edu.cn to the article.

The original article has been corrected.

Publisher's Note Springer Nature remains neutral with regard to jurisdictional claims in published maps and institutional affiliations

\footnotetext{
${ }^{1}$ HEC Research and Development Center, HEC Pharm Group, Dongguan, 523871, China.

${ }^{2}$ Hunan Provincial Key Laboratory of Xiangnan Rare-Precious Metals Compounds and Applications, College of Chemical Biology and Environmental Engineering, Xiangnan University, Chenzhou, 423043, China.

${ }^{3}$ College of Pharmacy, Yanbian University, Yanji, 133002, China.

${ }^{4}$ College of Pharmacy, Jinan University, 601 Huangpu Avenue West, Guangzhou, 510632, China.

${ }^{5}$ To whom correspondence should be addressed. (e-mail: ChenYong@hec.cn; quanguilan@jnu.edu.cn)
} 